\title{
Electrical Properties of Bi-Doped PbTe Layers Grown by Molecular Beam Epitaxy on $\mathrm{BaF}_{2}$ Substrates
}

\author{
A.M.P. dos Anjos, E. Abramof, P.H.O. Rappl, A.Y. Ueta, and H. Closs \\ Laboratório Associado de Sensores e Materiais - LAS, Instituto Nacional de Pesquisas Espaciais - INPE, \\ CP515, 12245-970, São José dos Campos-SP, Brazil
}

Received on 31 March, 2003

\begin{abstract}
Resisistivity and Hall measurements were performed at temperatures from 10 to $320 \mathrm{~K}$ on Bi-doped PbTe layers grown on (111) $\mathrm{BaF}_{2}$ by molecular beam epitaxy. Samples with electron concentration varying from $1 \times 10^{17}$ to $4 \times 10^{19} \mathrm{~cm}^{-3}$ were obtained. Results indicated that all offered $\mathrm{Bi}$ atoms in the vapor phase were effectively incorporated in the PbTe as active donors. No thermal activation in the whole doping range was observed, indicating that the $\mathrm{Bi}$ donor level lies resonant with the conduction band. The mobility curve showed that the $\mathrm{PbTe}$ layers tend to a metallic behavior as the electron concentration increases. A value around $1 \times 10^{19} \mathrm{~cm}^{-3}$ is suggested for $n^{+} \mathrm{PbTe}$ contact layers in device application.
\end{abstract}

\section{Introduction}

The control of the electron concentration in PbTe layers is very desirable when device fabrication is concerned. In order to accomplish optimization of electrical performance, different carrier concentrations are needed in the several layers that compose the device. The fabrication of a resonant tunneling double-barrier diode, for instance, requires low $n$ type $\mathrm{PbTe}$ near the barriers and high doping $\left(n^{+}\right)$in the bottom and top layers [1], and junctions like $p n^{+}\left(\right.$or $\left.n^{+} p\right)$ are interesting for infrared detector application [2,3]. Bismuth is normally used as an $n$-type dopant in PbTe layers, and is usually evaporated from $\mathrm{a} \mathrm{Bi}_{2} \mathrm{Te}_{3}$ source in a molecular beam epitaxy (MBE) system [4].

In this paper, we investigate the electrical properties of Bi-doped PbTe layers grown on (111) $\mathrm{BaF}_{2}$ substrates. Resistivity, carrier concentration, and Hall mobility were measured as a function of temperature for samples with different doping levels. The temperature dependent data allowed conclusions about the $\mathrm{Bi}$ impurity behavior in $\mathrm{PbTe}$ and the results supplied important information for the choice of the best doping condition for some device application.

\section{Experimental}

The PbTe layers were grown on freshly cleaved (111) $\mathrm{BaF}_{2}$ substrates by molecular beam epitaxy in a RIBER 32P MBE system equipped with effusion cells containing solid sources of $\mathrm{PbTe}, \mathrm{Te}$ and $\mathrm{Bi}_{2} \mathrm{Te}_{3}$. The beam flux from each cell was measured separately in an ion-gauge flux monitor. During this experiment, the $\mathrm{PbTe}$ and $\mathrm{Te}$ effusion cell temperatures were $656^{\circ} \mathrm{C}$ and $236^{\circ} \mathrm{C}$, leading to beam fluxes of $7.2 \times 10^{-7}$ and $1.3 \times 10^{-8}$ Torr, respectively. The layers were always deposited at a substrate temperature of $300^{\circ} \mathrm{C}$. In these conditions the growth rate was $2.2 \AA / \mathrm{s}$. In order to obtain the PbTe layers with different $\mathrm{Bi}$ doping levels, a series of samples were grown with $\mathrm{Bi}_{2} \mathrm{Te}_{3}$ effusion cell temperatures varying from 360 to $450^{\circ} \mathrm{C}$.

For the electrical characterization, Van der Pauw geometry samples were prepared by soldering $\mathrm{Au}$ wires with In pellets. The samples were mounted in Ni-coated $\mathrm{Cu}$-plates and assembled in a He closed-cycle cryostat equipped with an auto-tune temperature controller. The electrical measurements were performed in a Keithley Hall effect system with a magnetic field of 7000 Gauss. All equipments were computer controlled via an IEEE-488 interface. The control and data acquisition program, which allows automatic temperature dependent measurements, was developed in a Visual Basic platform.

\section{Results and discussion}

Resistivity and Hall effect were measured on the PbTe layers grown at different doping levels at temperatures from 10 to $320 \mathrm{~K}$. Fig. 1 plots the electron concentration at $77 \mathrm{~K}$ as a function of the $\mathrm{Bi}_{2} \mathrm{Te}_{3}$ effusion cell temperature. The electron concentration follows approximately the same exponential temperature dependence of the $\mathrm{Bi}_{2} \mathrm{Te}_{3}$ vapor pressure, indicating that, at this temperature range, the offered $\mathrm{Bi}$ atoms in the vapor phase are being effectively incorporated in the PbTe as active donors.

Normally, in the lead salts, the native defects created by the deviation from stoichiometry are electrically active, i.e. metal or chalcogen vacancies act as acceptors or donors, respectively. The levels created by the vacancies are resonant with the valence and conduction bands, and no carrier freeze out is observed at low temperatures. Contrary to the semiconductor materials with impurity levels within the energy 
gap, the thermal activation of majority carriers is not observed in the undoped lead salts. For the case of the Bidoped layers investigated here, the temperature dependence of the electron concentration did not exhibit any type of thermal activation in the whole doping range, as demonstrated in Fig. 2. This fact indicates that the doping level introduced by the $\mathrm{Bi}$ atoms also lies resonant with the conduction band.

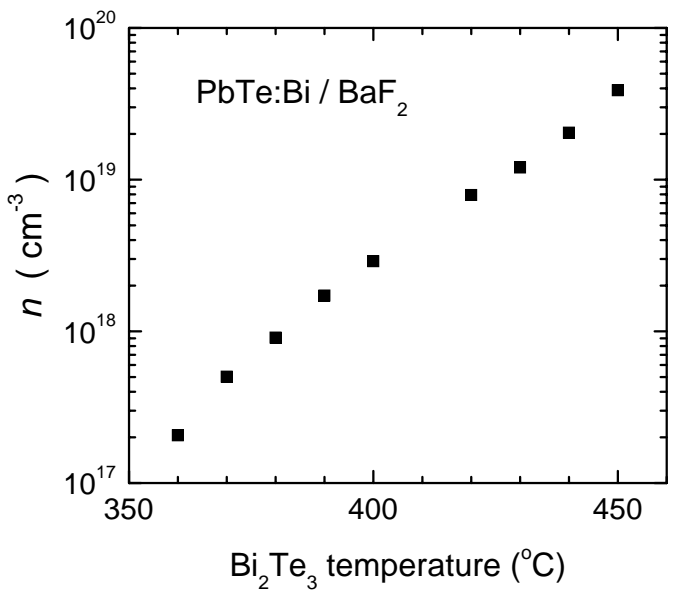

Figure 1. Electron concentration at $77 \mathrm{~K}$ of $\mathrm{Bi}$-doped $\mathrm{PbTe}$ layers as a function of the $\mathrm{Bi}_{2} \mathrm{Te}_{3}$ effusion cell temperature.

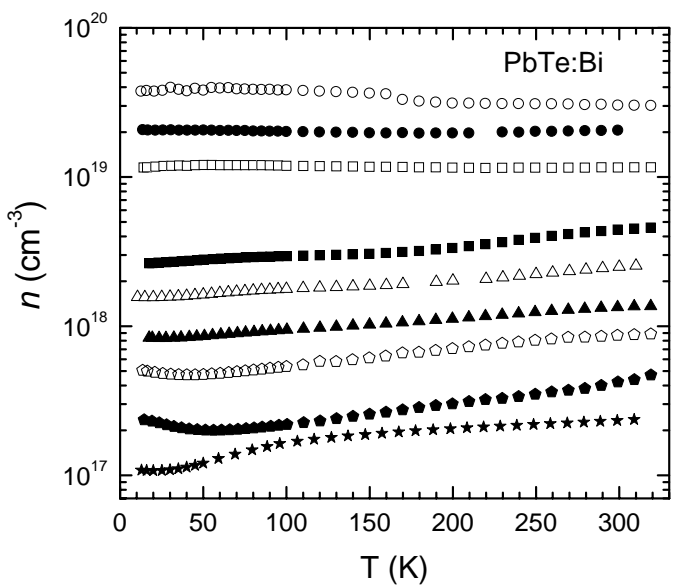

Figure 2. Electron concentration versus temperature of PbTe layers doped with bismuth. The stars are data from an undoped $n$-type layer.

Since the carrier concentration almost does not change with temperature, the mobility in the lead salts is, in general, determined by the phonon scattering at temperatures higher than $80 \mathrm{~K}$. At low temperatures, the carrier scattering due to ionized impurities, which is normally observed for the most common semiconductor materials, is not present in the IV-VI compounds. The huge static dielectric constant, which screens the Coulomb potential of the impurities, and the relatively small effective masses are responsible for the high mobilities observed in PbTe at low temperatures. If well-controlled additional Te flux and elevated growth temperatures are used, low-temperature saturation mobility of above $10^{6} \mathrm{~cm}^{2} / \mathrm{V}$.s is obtained in $n$-PbTe [5]. The mobility saturation at low temperatures is related to carrier scattering due to defects, mainly dislocations.

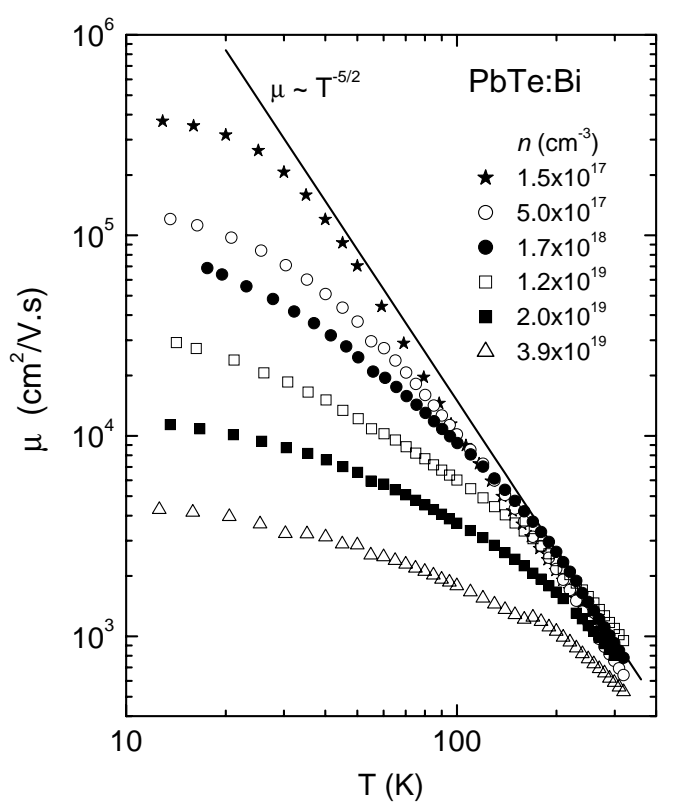

Figure 3. Hall Mobility versus temperature of $\mathrm{PbTe}: \mathrm{Bi}$ samples with increasing electron concentration. Stars data belong to an undoped sample.

Figure 3 shows the Hall mobility as a function of temperature for the PbTe layers with increasing Bi doping level. The data for an undoped $n$-type PbTe layer is also included. It is important to emphasize that the MBE growth conditions for the undoped and Bi-doped samples used in this work were not optimized to achieve the highest saturation mobility. Nevertheless, general features of the mobility curve as a function of electron concentration can be evidenced. Although a pure metal-like PbTe is not observed in this set of samples, the PbTe layers tend to a metallic behavior as the electron concentration increases. The phonon scattering limiting behavior $\left(\mu \sim \mathrm{T}^{-5 / 2}\right)$, represented by the solid line in the graph, is observed at $T>100 \mathrm{~K}$ for PbTe layers with $n$ values up to $1 \times 10^{18} \mathrm{~cm}^{-3}$. For higher electron concentrations, the mobility starts to deviate from this limit earlier at higher temperatures. At room temperature, where phonons dominate over other scattering mechanisms, the mobility shows a small decrease from 900 to $500 \mathrm{~cm}^{2} / \mathrm{V}$.s, as the doping concentration increases from $1.5 \times 10^{17}$ to $3.9 \times 10^{19} \mathrm{~cm}^{-3}$. At an intermediate temperature of $80 \mathrm{~K}$, this reduction becomes more evident (27000 to 2100 $\mathrm{cm}^{2} /$ V.s), indicating that, at this temperature range, additional carrier scattering processes start to limit the mobility as the carrier concentration increases. The low-temperature saturation mobility has the most pronounced reduction with electron concentration, as expected. It decreases from $4 \times 10^{5}$ $\mathrm{cm}^{2} / \mathrm{V}$.s, for the undoped sample, to $4 \times 10^{3} \mathrm{~cm}^{2} / \mathrm{V}$.s, for the sample with $n=3.9 \times 10^{19} \mathrm{~cm}^{-3}$. 


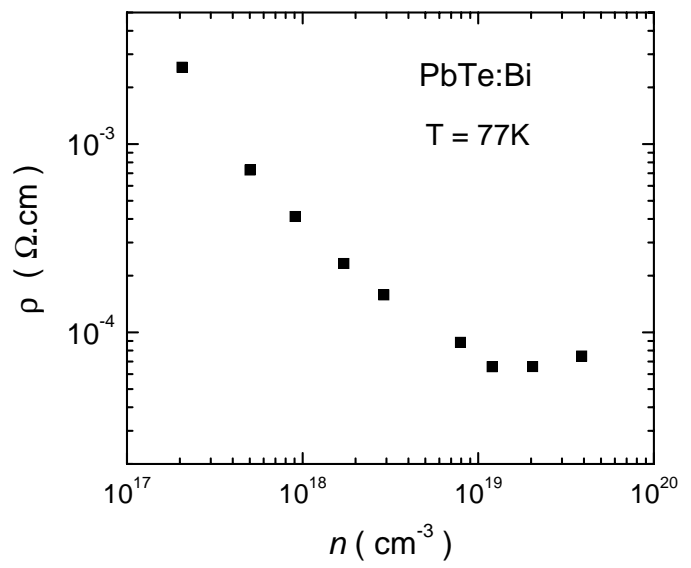

Figure 4. Resistivity as a function of electron concentration in $\mathrm{PbTe}$ layers with different doping levels.

In order to test the influence of the doping process on the crystalline quality of the samples, $\mathrm{x}$-ray rocking curves were measured around the (222) Bragg diffraction peak of all samples. The full-width at half-maximum varied randomly from 70 to 140 arcsec, showing no influence of the $\mathrm{Bi}$ doping on the dislocation density in the PbTe layers. This result indicates that the pronounced reduction of mobility at low temperatures is only due to the substantial increase of the electron concentration.
To find the best values for $n^{+}$PbTe contact layers, the resistivity at $77 \mathrm{~K}$ (normal device operation temperature) is plotted in Fig. 4 as a function of electron concentration.

The resistivity decreases from approximately $3 \times 10^{-3}$ to $6 \times 10^{-5} \Omega . c \mathrm{~m}$ as the electron density increases from $1 \times 10^{17}$ to $1 \times 10^{19} \mathrm{~cm}^{-3}$, and starts to rise slowly as more $\mathrm{Bi}$ atoms are incorporated. This result suggests a value around $1 \times 10^{19} \mathrm{~cm}^{-3}$ for the $n^{+}-\mathrm{PbTe}$ contact layers in device application.

\section{Acknowledgement}

We are grateful to partial support of FAPESP (Grant Nr. 00/12529-6) and CAPES (scholarship of first author).

\section{References}

[1] P. Han, M. Sakuraba, Y.-C. Jeong, K. Bock, T. Matsuura, and J. Murota, J. Cryst. Growth 209, 315 (2000).

[2] J. John and H. Zogg, J. Appl. Phys. 85, 3364 (1999).

[3] C. Boschetti, P.H.O Rappl, A.Y. Ueta, and I.N. Bandeira, Infrared Phys. 34, 281 (1993).

[4] A. Prinz, G. Springholz, A.Y. Ueta, and G. Bauer, Phys. Rev. B 59, 12983 (1999).

[5] G. Springholz, G. Bauer, and G. Ihninger, J. Cryst. Growth 127, 302 (1993). 